\title{
Practical Achievable Performance in Diesel Oxidation Catalyst Temperature Control
}

\author{
O. Lepreux ${ }^{1 *}$, Y. Creff $^{1}$ and N. Petit ${ }^{2}$ \\ 1 IFP Energies nouvelles, Technology, Computer Science and Applied Mathematics Division, BP 3, 69360 Solaize - France \\ 2 MINES ParisTech, Centre Automatique et Systèmes, Mathématiques et Systèmes, 60 bd Saint-Michel, 75272 Paris Cedex 06 - France \\ e-mail: olivier.lepreux@ifpen.fr - yann.creff@ifpen.fr - nicolas.petit@mines-paristech.fr \\ * Corresponding author
}

\begin{abstract}
Résumé - Performances atteignables en pratique pour la commande de la température d'un catalyseur d'oxydation Diesel - Ce papier propose un modèle simplifié pour la commande d'un catalyseur d'oxydation Diesel (DOC). Ce modèle à paramètres répartis prend en compte la génération de chaleur spatialement répartie liée à l'oxydation des réducteurs. Il vise à être utilisé pour la commande de la température en sortie du DOC. Cette commande est nécessaire lors des phases de régénération du filtre à particules (DPF). Le papier s'intéresse en particulier aux phénomènes impliqués par les variations de débit de gaz dans le DOC. Ces variations ont lieu pendant les changements de points de fonctionnement du moteur liés à la demande du conducteur : elles sont importantes et fréquentes et ne peuvent pas être évitées. Les résultats expérimentaux présentés dans ce papier montrent qu'une augmentation du débit de gaz - à concentration $\mathrm{HC}$ constante en entrée du DOC - a tendance à créer un dépassement positif de la température en sortie du DOC. Au contraire, une diminution du débit a tendance à créer un dépassement négatif de la température en sortie du DOC. L'utilisation d'une mesure de température au milieu du pain catalytique permet de montrer que ces perturbations sont inhérentes à la nature répartie du système. La compensation de ces variations de température en sortie est physiquement limitée (par les limites d'actionnement et d'utilisation raisonnable du système), ce qui crée une perturbation retardée de la température en sortie du DOC. En conséquence, il semble qu'il existe une limite pratique pour les performances de commande de la température en sortie du DOC, qui sont, pour le DOC présenté (4 pouces de long et $1,65 \mathrm{~L}$ de volume), d'environ $+/-15^{\circ} \mathrm{C}$ autour de la température de sortie souhaitée. Le modèle proposé, sa validation expérimentale, ainsi que les analyses des performances obtenues sur un banc d'essai constituent les contributions principales de cet article.
\end{abstract}

\begin{abstract}
Practical Achievable Performance in Diesel Oxidation Catalyst Temperature Control This paper proposes a simple control-oriented model for a Diesel Oxidation Catalyst (DOC). This distributed parameter model accounts for spatially distributed heat generation due to oxidation of reductants. It aims at being used in the context of DOC outlet temperature control, which is required during Diesel Particulate Filter (DPF) active regeneration process. The paper focuses particularly on phenomena involved during variations of gas flow rate through the DOC. These variations take place during changes of engine operating points due to driver's requests: they are large and frequent, and cannot be avoided. Experimental results presented in this paper show that an increase in the gas flow rate, at constant inlet HC concentration, tends to make DOC outlet temperature overshoot. On the contrary, a decrease in the gas flow rate tends to make DOC outlet temperature undershoot. Using an intra-catalyst measurement, it is shown that these disturbances are related to the inherent distributed
\end{abstract}


nature of the system. Compensating for observed outlet temperature variations is physically limited (by actuation limits and reasonable use of the system) and creates a delayed DOC outlet temperature disturbance. As a consequence, it seems that there exists a practical limit for the DOC outlet temperature control performance, which is, for the presented 4-inch long $(1.65-\mathrm{L}) \mathrm{DOC}$, approximately $\pm 15^{\circ} \mathrm{C}$ around the desired outlet temperature. The proposed simple model, its experimental validation, and the obtained analysis of performance based on testbench results are the main contributions of the article.

\section{INTRODUCTION}

On most modern Diesel vehicles, increasingly stringent particulate matter emissions [1] are satisfied using a Diesel Particulate Filter (DPF). This filter, located in the vehicle exhaust line, stores particulate matter until it is burnt in an active regeneration process [2]. During this phase, DPFs behave like potentially unstable reactors [3], and their inlet temperature must be carefully controlled to prevent filter runaway.

In most current aftertreatment architectures [4], a Diesel Oxidation Catalyst (DOC) is placed upstream of the DPF in the vehicle exhaust line. To increase the DPF inlet temperature, reductants are oxidized in the DOC, which, in turn, increases its outlet temperature. The DOC also conveys, up to some heat losses, its inlet heat flow: in other words, inlet temperature variations propagate through the DOC.

A DOC is a chemical system difficult to control. Classical models are usually composed of a dozen of coupled Partial Differential Equations (PDEs) [5], which complicates the development of model-based control strategies. Experimentally, it can be observed that a step change in the inlet temperature propagates to the output of the system with long response times [6]. Depending on the engine outlet gas flow rate, these response times significantly vary: they roughly decrease by a factor of 10 from idle speed to full load. Strategies that are commonly used to deal with this problem rely on look-up tables, which, in practice, are difficult and time-consuming to calibrate.

The purpose of this paper is to investigate phenomena involved in the DOC during gas flow variations, to show their consequences on the outlet temperature, and on the achievable control performance. A simple DOC controloriented model is proposed, which aims at being used during DPF regeneration process (adaptations could be required to deal with the issue of catalyst ageing, which is not treated in this paper).

After a general introduction, a very simple macroscopic model is recalled (presented in [7]) to describe temperature evolution in a DOC. Then, DOC temperature evolution under variable gas flow conditions is described and the corresponding model is validated. Finally, the possibilities of compensation are investigated and conclusions are drawn about the temperature control performance.

\section{DOC MODELING}

In this section, after a brief presentation of the thermal phenomena involved in the DOC at different flow rates, a model is proposed to account for the control by reductant mass flow rate and validated at different constant gas flow conditions. The proposed simple model, its experimental validation, and the obtained analysis of performance based on testbench results are the main contributions of the article.

\subsection{Physical System and Preliminary Observations}

The system studied in this paper is a DOC as present in the vehicle exhaust line. An example of configuration of aftertreatment devices in the exhaust line is pictured in Figure 1. Other devices may be present in the exhaust line but the problem exposed in this paper remains roughly the same.

The DOC is composed of numerous thin channels conveying the gas flow. This geometry implies efficient heat exchange between gas and monolith. Gas/solid exchanges are governing thermal phenomena described in most catalyst models (see e.g. [5]), and will be used in Section 1.2. Because of this, rise time can be longer than a hundred seconds while the gas flows through the DOC in a fraction of a second. Figure 2 illustrates these phenomena. As detailed in previous publications [7-10], inlet temperature variations $\left(T^{\text {in }}\right)$ and HC variations imply highly-delayed outlet temperature $\left(T^{\text {out }}\right)$ responses. Time constant and delay of these responses are mostly dependent on the gas flow rate (see [11] for details about the identification process to this delayed first order system), as plotted in Figure 3.

At first order, HC conversion efficiency of the DOC defined as the ratio of consumed $\mathrm{HC}$ to inlet $\mathrm{HC}$ - depends on the gas flow rate [11]. As presented in Figure 4, at low

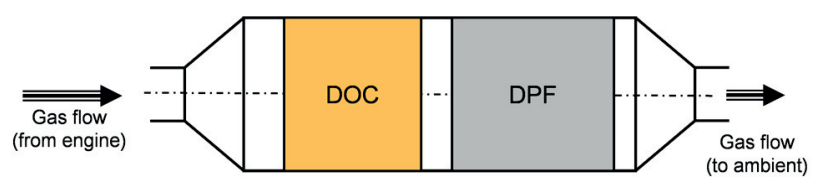

Figure 1

Example of a possible configuration of aftertreatment devices in the exhaust line. 


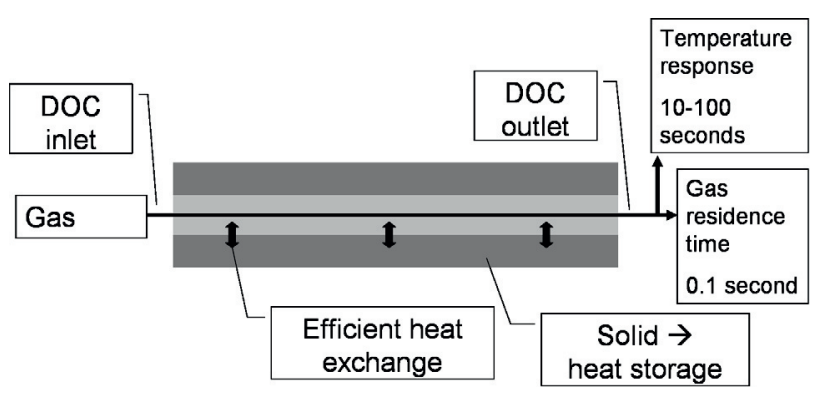

Figure 2

DOC phenomena for temperature delay involved in the outlet temperature dynamics.
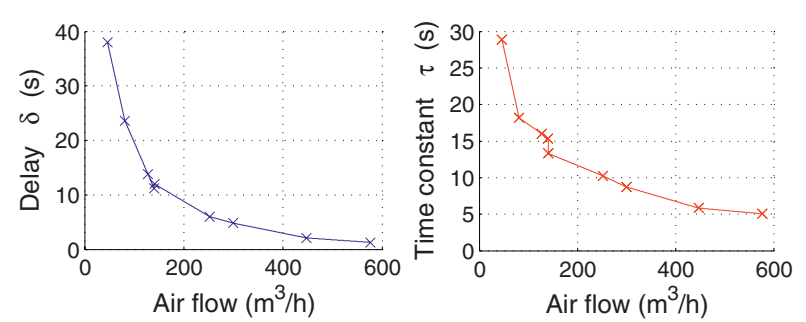

Figure 3

Evolution of $\delta$ and $\tau$ versus gas flow rate (experimental data).

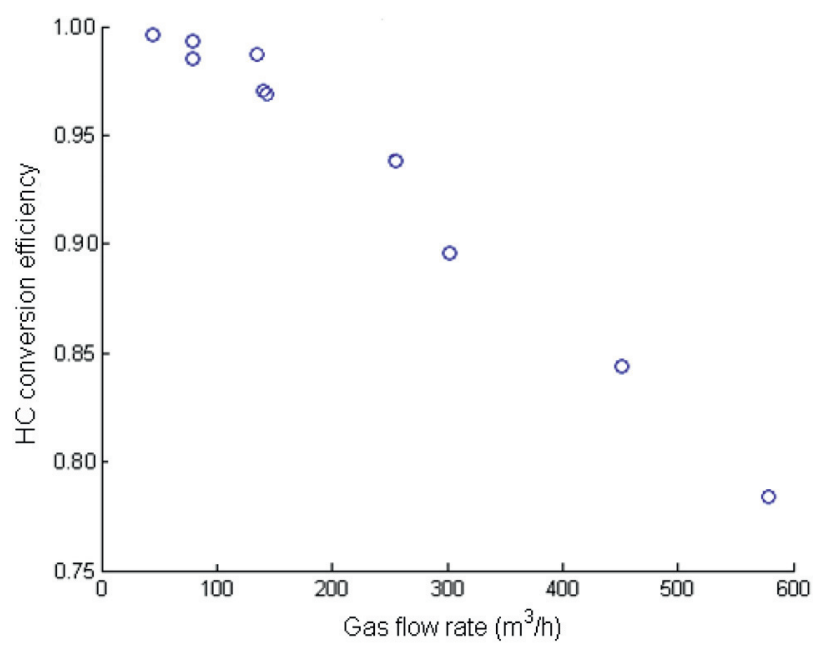

Figure 4

DOC efficiency versus mass gas flow rate (experimental data on a 3-inch long DOC).

gas flow rate, the efficiency is very high. It decreases continuously with increasing gas flow rate. These results are obtained at high temperature and under lean conditions. It is important to note that in case of a very high concentration of reductant, the efficiency of the DOC decreases and cannot be considered as a function of the gas flow rate only.

Finally, a transient phenomenon, which motivates discussions in this paper, is presented in Figure 5. In this
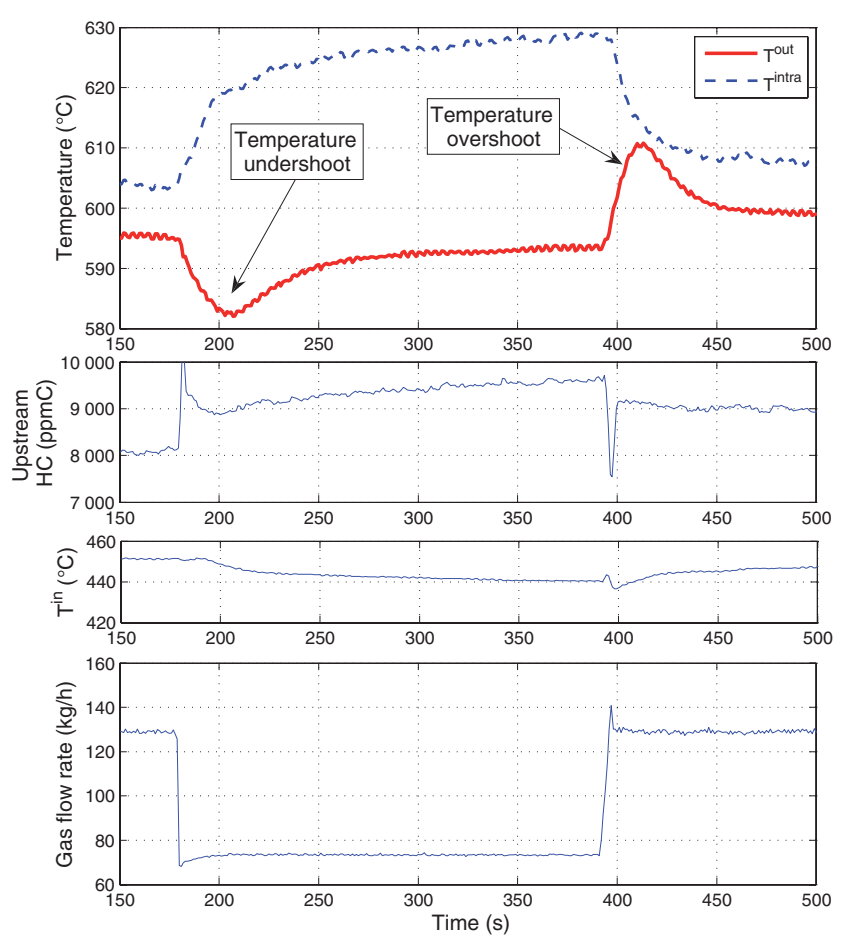

Figure 5

Inverse phenomena (experimental data). HC actuation is controlled by static mapping, which ensures the synchronization to the operating point. HC peaks are voluntarily generated to ensure unfavorable conditions for these phenomena. Dead time of the $\mathrm{HC}$ analyzer is accounted for in this figure.

experiment, it is desired to keep the output temperature constant during a gas flow rate variation. Pseudo-steadystate input conditions are used. It can be observed that the output temperature transiently overshoots above or under its steady-state level. This transient phenomenon - later denoted "overshoot" or "undershoot", phenomenon - is discussed more specifically in Section 2.

\subsection{Model with Heat Source}

In [8], a simple control model is proposed. It is shown that step responses of the system can be identified to this model with good quality. However, its representation might seem a bit simplistic in view of real applications since inlet temperature variations $T^{\text {in }}$ (which play the key role of input in this model) are difficult to control and cannot be used directly as control variable. In practice, reductants (hydrocarbons HC) are injected at the inlet of the DOC (control variable $u$ ). They are oxidized on the catalyst and, consequently, increase the DOC temperature $T^{\text {out }}$. The system studied in this paper and corresponding input and output variables are pictured in Figure 6, where $v$ is the gas speed (straightforwardly related to the gas flow rate). The corresponding model is described hereafter. 


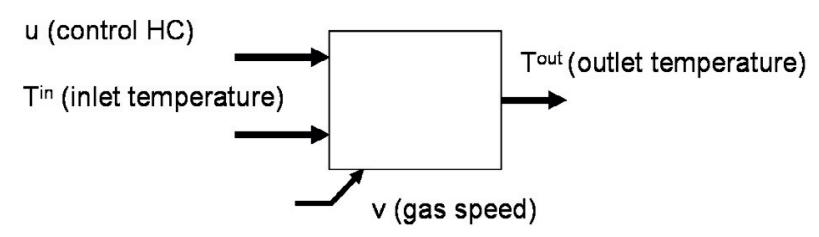

Figure 6

DOC system.

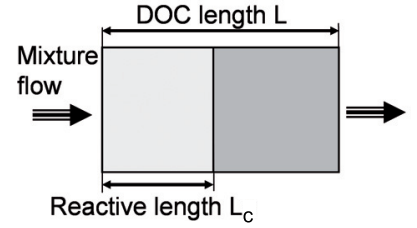

Figure 7

$\mathrm{HC}$ reaction zone.
During the regeneration process, the DOC is working at high temperatures, which ensures that the rate of conversion of reductants is high. Moreover, a large quantity of $\mathrm{HC}$ is injected to produce heat. Consequently, the effect of this reductant is dominating over other species'. For these reasons, to model the DOC thermal behavior, it is proposed to encompass all the chemical reactions in a "source term $\xi$ ", leading to the following control-oriented model

$$
\left\{\begin{array}{l}
\frac{\partial T}{\partial t}(z, t)+v \frac{\partial T}{\partial z}(z, t)=-k_{1}\left(T(z, t)-T_{s}(z, t)\right) \\
\frac{\partial T_{s}}{\partial t}(z, t)=k_{2}\left(T(z, t)-T_{s}(z, t)\right)+\xi(z, t)
\end{array}\right.
$$

where $T$ and $T_{s}$ are respectively gas and solid temperatures, $v$ is the channel gas speed which can be derived from mass flow, parameters $\left(k_{1}, k_{2}\right)$ can be either derived from usual correlations [12] or identified from experimental data [8]. The output of the system is the outlet gas temperature $T^{\text {out }}(t) \triangleq T(L, t) . T^{\text {in }}(t) \triangleq T(0, t)$ is regarded as a disturbance and $\xi(z, t)$ will be considered as an input variable. $\xi$ is a lumped variable, including in particular the sum of the enthalpies of the various reactions taking place inside the DOC.

As mentioned above, the catalyst is used at a temperature significantly higher than the light-off temperature. Experimental HC step responses are not really impacted by the temperature level. Implicitly, it is considered that the temperature does not influence the source term $(\xi(z, t)$ is not a function of $T_{S}$ ). By construction, a DOC is designed to yield large heat and mass transfer. These transfers are very effective, and the time constants implying the thermal phenomena are much larger than the ones implying chemical reactions. For the experiments presented in Section 1.4, gas flows through the DOC approximatively 1000 times faster than the outlet temperature response time. Because there is no $\mathrm{HC}$ storage effect at high temperature, and because experiments show high conversion rates, time constants of chemical reactions are at least one order of magnitude lower than thermal time constants. Hence, for a thermal model, it can be assumed that the source term establishes very rapidly to its steady-state value, that may depend on the total amount of reductants and the gas speed. Hence, it is assumed that the source term $\xi$ can be approximated by a function denoted $\Psi(z, v(t), u(t))$, where $u$ is the control variable (injected reductants flow rate). Note that $\Psi(z, v(t), u(t))$ is not explicitly a function of $t$. Then, a strong simplifying assumption is formulated. Namely, $\Psi$ is assumed uniform over some spatial interval. This assumption is supported by experimental identification results of Section 1.4. Over the whole range of considered operating conditions, the obtained results are quite accurate. Denote $L_{c}$ the length of the portion of the DOC where the heat of reaction is released (see Fig. 7). Formally, the following discontinuous function is considered

$$
\begin{cases}\Psi(z, u(t), v(t))=\psi(u(t), v(t)), & \text { if } 0 \leq z \leq L_{c}(v(t)) \\ \Psi(z, u(t), v(t))=0, & \text { if } L_{c}(v(t))<z \leq L\end{cases}
$$

where $\psi$ is related to the control variable $u$.

In the following, the source term step response is calculated for constant $L_{c}$. It is used for constant gas speed conditions in Section 1.4 and Section 1.5. When dealing with variable gas speeds, as it is the case in Section 2.2, a spatial discretization of the model is used. Write $\hat{\psi}(s)=\alpha / s$, where $\alpha$ is a constant, $s$ is the Laplace variable, and $\hat{x}$ is the Laplace transform of $x$. Then, several steps of operational calculus in Equation (1) lead to

$$
\begin{aligned}
& \hat{T}\left(L_{c}, s\right)=\hat{T}^{i n} \exp \left(-\hat{A}(s) L_{c}\right) \\
& +\frac{\hat{B}(s)}{\hat{A}(s)}\left(1-\exp \left(-\hat{A}(s) L_{c}\right)\right)
\end{aligned}
$$

with $\hat{A}(s)=\frac{1}{v}\left(s+k_{1}-\frac{k_{1} k_{2}}{s+k_{2}}\right)$ and $\hat{B}(s)=\frac{k_{1}}{v} \frac{\alpha}{s\left(s+k_{2}\right)}$. In Equation (3), the first term corresponds to the transfer from the inlet temperature $T^{i n}(t)$ to the output $T\left(L_{c}, t\right)$, while the second term corresponds to the transfer from the input signal $\hat{\Psi}$ defined in Equation (2) to the output $T\left(L_{c}, t\right)$. Further, for $z>L_{c}$, Equation (1) gives $\hat{T}(z, s)=\hat{T}\left(L_{c}, s\right) \exp \left(-\hat{A}\left(z-L_{c}\right)\right)$ and

$$
\begin{gathered}
\hat{T}(z, s)=\hat{T}^{i n} \exp (-\hat{A} z)-\frac{\hat{B}}{\hat{A}} \exp (-\hat{A} z) \\
+\frac{\hat{B}}{\hat{A}} \exp \left(-\hat{A}\left(z-L_{c}\right)\right)
\end{gathered}
$$


Eventually, by an inverse Laplace transform of Equation (4) (detailled in [13]), one obtains the reductant step response

$$
\begin{aligned}
& T(z, t)=\Upsilon(t-z / v) \exp \left(-\frac{k_{1} z}{v}\right) M(z, t-z / v) \\
& -\Upsilon(t-z / v) \exp \left(-\frac{k_{1} z}{v}\right) F(z, t-z / v) \\
& +\Upsilon(t-z / v) \exp \left(-\frac{k_{1} z}{v}\right) F\left(z-L_{c}, t-z / v\right)
\end{aligned}
$$

where $\Upsilon$ is the Heaviside step function and

$$
\left\{\begin{array}{l}
M(z, t)=T^{i n}(t) \\
+\int_{0}^{t} \exp \left(-k_{2} \tau\right) \sqrt{\frac{m(z)}{\tau}} \mathrm{I}_{1}(2 \sqrt{m(z) \tau}) T^{i n}(t-\tau) d \tau \\
F(z, t)=g(t) \\
\quad+\int_{0}^{t} \exp \left(-k_{2} \tau\right) \sqrt{\frac{m(z)}{\tau}} \mathrm{I}_{1}(2 \sqrt{m(z) \tau}) g(t-\tau) d \tau \\
g(t)=\frac{k_{1} \alpha}{k_{1}+k_{2}} t-\frac{k_{1} \alpha}{\left(k_{1}+k_{2}\right)^{2}}\left(1-\exp \left(-\left(k_{1}+k_{2}\right) t\right)\right) \\
m(z)=k_{1} k_{2} z / v
\end{array}\right.
$$

with $\mathrm{I}_{1}$ the modified Bessel function of the first kind.

The steady state value of the response of $T^{\text {out }}$ to the step variation $\psi$ can be computed using Equation (4)

$$
\begin{aligned}
& \lim _{t \rightarrow \infty} T(L, t)=\lim _{s \rightarrow 0} s \hat{T}(L, s) \\
& =\lim _{s \rightarrow 0}\left[s \frac{\hat{B}}{\hat{A}}\left(1-\hat{A}\left(L-L_{c}\right)\right)-s \frac{\hat{B}}{\hat{A}}(1-\hat{A} L)\right] \\
& =\lim _{s \rightarrow 0} s \hat{B} L_{c}=\frac{k_{1} \alpha L_{c}}{k_{2} v}
\end{aligned}
$$

$T^{i n}$ is considered constant for the computation. However, if $T^{\text {in }}$ is varied together with $\psi$, its contribution is simply added to the steady state value of Equation (6). Formula (6) is used during the identification and normalization process in this section. In practice, it is possible to relate $\alpha, L_{c}$, the current $\mathrm{HC}$ conversion efficiency $\eta_{H C}$ and the amount of injected reductants $u$ (which is itself related to the injector energizing time): for a given (identified) $L_{c}, \alpha$ can be regarded as a control variable as it is proportional to $u$.

\subsection{Experimental Setup}

The experimental setup is as follows. The DOC is located in a Diesel engine exhaust line. The OEM ECU is fully bypassed. The engine is used to control the gas flow rate and the DOC inlet temperature. Two distinct configurations are used to control the reductant flow rate: either by an additional injector located right upstream of the turbine or by common-rail late post-injection. It is worth mentioning that the HCs generated by these two injection methods are different and can actually affect DOC conversion efficiency [11]. This effect can be significant at some operating points. Values obtained in this paper depend on each particular tested

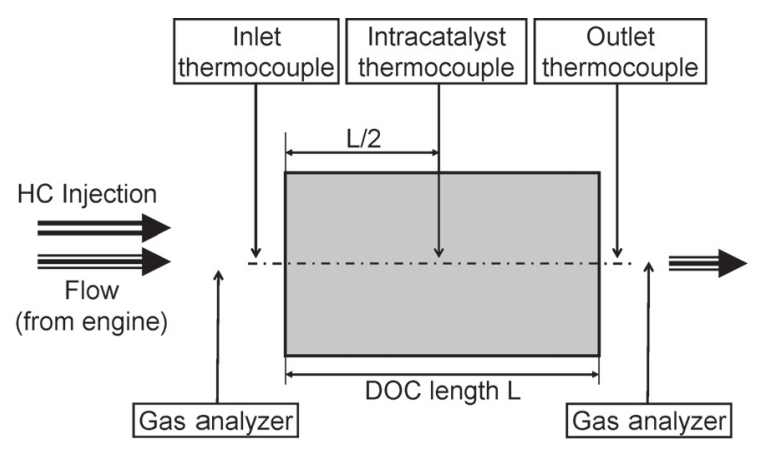

Figure 8

Experimental setup.

system. Temperature is measured at three different locations and gas is analyzed upstream or downstream of the DOC by a 5-gas bench (as presented in Fig. 8). In some experiments, gas analyses are reported for both upstream and downstream locations. In fact, these experiments have been carried out twice, taking a particular care to guarantee that all external conditions, inputs and measurements were kept very similar. DOC inlet temperature $T^{\text {in }}$ is raised from $300^{\circ} \mathrm{C}$ to $500^{\circ} \mathrm{C}$ by the mean of a coupled post-injection in addition to the pilot and the main injections. Presented experimental results are obtained using two distinct engine and DOC setups. Two 2.2-L 4-cylinder engines are used. A 3-inch long and a 4inch long platinum-based DOC are tested. Both the monoliths are made of cordierite and have a cell density of 400 cpsi.

Considering those configurations, generating a disturbance (engine emissions, $T^{\text {in }}$, gas flow rate) independently from the others is not possible. This explains why they are not decoupled in the results presented in Section 2.

\subsection{Experimental Validation of HC Step Responses}

Figure 9 presents experimental HC step responses under various operating conditions. It is shown that the model (1) with input (2) fits experimental data well.

\subsection{Effects of Inlet Temperature}

From this model, it is shown in Figure 10, that the $T^{\text {in }}$ response is slower than the reductant response. The $T^{\text {in }}$ effect is highly delayed because of phenomena described in Section 1.1. This is an important result because it shows that $T^{\text {in }}$ does not have a direct influence on the overshoot phenomena that will be presented in Section 2 .

\subsection{Effects of Variation of Reactive Length}

In this subsection, consequences of a change in the reactive length are explained. It has been introduced that $\mathrm{HC}$ combustion is considered to take place in a zone at the beginning 


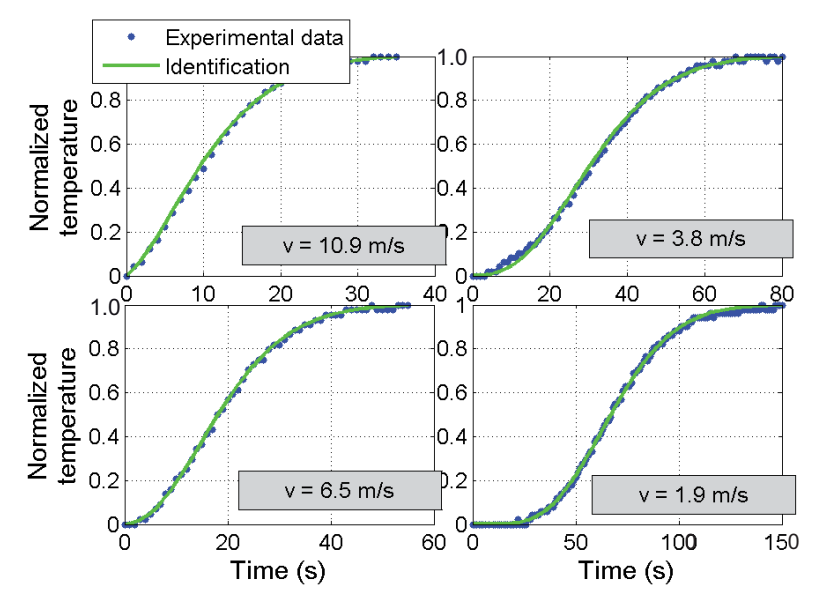

Figure 9

Experimental HC step response in various operating conditions and at constant gas flow rate. Part of the fitting set.

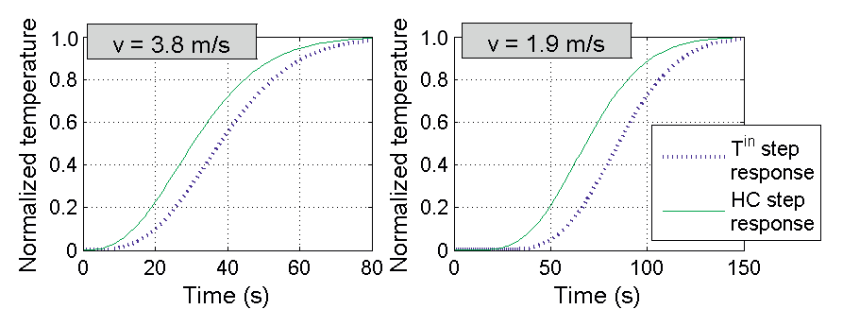

Figure 10

Comparison between HC step response and $T^{\text {in }}$ step response. Analytic results obtained from Equation (5).

of the DOC. Again, it is worth noticing that adaptations could be required to account for a deteriorated zone due to catalyst ageing. The length of this zone strongly depends on the catalyst and is a priori unknown. Additionally, for a given catalyst, it can vary depending on the operating point. From the adapted macroscopic viewpoint, the heat of reaction is homogeneously released on the reactive length and is zero on the downstream "transport" length. In the "transport" zone, the stored heat is such that the outlet temperature remains at a given level. Now, consider an increase in the reactive length as pictured in Figure 11a. During this transient, a part of the former transport zone is covered by the reactive zone, where $\mathrm{HC}$ reaction heat is now released. So, the new heat supply in this covered zone is more than required and causes an outlet temperature increase until steady state is reached. Now, consider a decrease in the reactive length: there exists a zone which is not supplied with heat during the transient. This causes an outlet temperature decrease until steady state is reached.

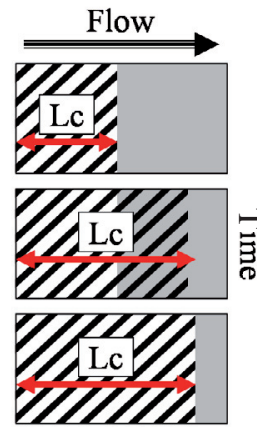

(a) Increase in Lc

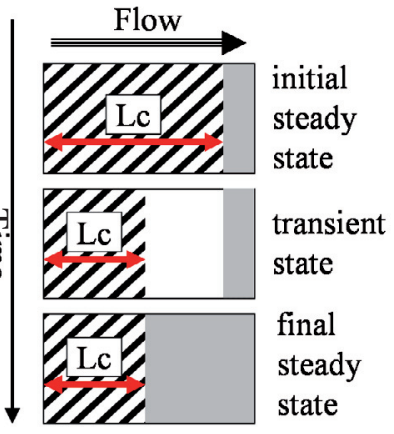

(b) Decrease in Lc
Figure 11

Phenomena involved during a variation of reactive length.

\subsection{Model Description}

It is proposed here to detail the dependency of the reactive length $L_{c}$ of model (1) upon gas flow rate. As a first approximation, $L_{c}$ is considered to be a piecewise affine function of the channel gas speed $v$,

$$
L_{c}=\min (L, a \cdot v+b)
$$

where $a$ and $b$ are positive constants, and $L$ is the DOC length. Typical values, for example on a 3 -inch long DOC system, are $a=7.6 \cdot 10^{-3} \mathrm{~s}$ and $b=3.7 \cdot 10^{-2} \mathrm{~m}$. This model expresses, in a very simple way, that likelihood for $\mathrm{HC}$ molecules to reach the catalytic surface increases with gas residence time in the monolith. Saturation of $L_{c}$ to $L$ is assumed for obvious physical reasons. It is corroborated by identification results, obtained in Section 1.4. An implicit assumption of this model is that $L_{c}$ and $u$ independent. In particular, the reactive length remains unchanged when $\mathrm{HC}$ inlet concentration is changed. It will be seen later in Section 2.4 that this assumption has some practical limits. However, it will be shown in the next section that this simple model is able to describe in good details transient experiments (obtained with the two tested devices) under DPF regeneration conditions.

\section{TEMPERATURE RESPONSE TO VARIABLE GAS FLOW CONDITIONS}

In Section 1.4 the DOC outlet temperature response to $\mathrm{HC}$ variations has been studied. It has been shown that identification to experimental data yields good results at constant gas flow rate. In this section, the DOC outlet temperature response under variable gas flow conditions is studied. First, the model presented in Section 1.7 is compared against experimental results. Then, the paper experimentally explores the possibilities to compensate for the outlet temperature variations caused by gas flow rate variations. 


\subsection{Effects of Gas Flow Rate Variations on the DOC Outlet Temperature}

As explained in Section 1.3, considering the experimental device, gas flow rate variations cannot be independent of inlet temperature variations nor of the engine emissions (especially $\mathrm{HC}$ and $\mathrm{CO}$ ).

It can be noticed in Figure 5 that a fall in gas flow rate (associated with an increase in efficiency as detailed in Sect. 1.1) and an increase in HC concentration upstream of the DOC imply an increase in the intra-catalyst temperature. Despite this increase, the outlet temperature is undershooting during a transient phase.

By contrast, an increase in gas flow rate (associated with a fall in efficiency) and a fall in HC concentration imply a fall in intra-catalyst temperature. Despite this decrease, the outlet temperature is overshooting during a transient phase.

Experimental results show that these two phenomena appear irrespective of whether the inlet temperature is rising or falling (see Fig. 15 for an example of a rising inlet temperature while the outlet temperature is undershooting). This is not surprising, because, as explained in Section 1.1 and Section 1.5, there is no direct transfer from the inlet temperature to the outlet temperature. Effects of the inlet temperature are highly delayed due to the storage effects along the monolith. On the contrary, the described phenomena are almost synchronous to the gas flow rate variations because they take place in the vicinity of the outlet of the DOC.

\subsection{Model Experimental Validation}

The phenomena caused by gas flow rate variations have been experimentally presented. Now, it is shown that those effects are captured by the proposed model.

The model presented in Section 1.7 is compared against experimental data in Figure 12. The disturbances (mass gas flow, inlet temperature, inlet HC) are also reported. The temperature variations are well represented by our model. In particular, it can be seen that the phenomena of "overshoot" and "undershoot" related to gas flow rate increase at $t=90 \mathrm{~s}$ and decrease at $t=275 \mathrm{~s}$. For comparison, the simulation results in the case of a constant reactive length are also plotted. This stresses how the variable reactive length affects outlet temperature during gas flow transients.

Now that it has been established that gas flow rate variations cause undesired outlet temperature evolutions, the next subsections study strategies of compensation.

\subsection{Compensation of Overshoot Phenomenon}

The overshoot phenomenon, which has been introduced in the previous subsections, is related to an increase in gas flow rate. This overshoot phenomenon is problematic in a
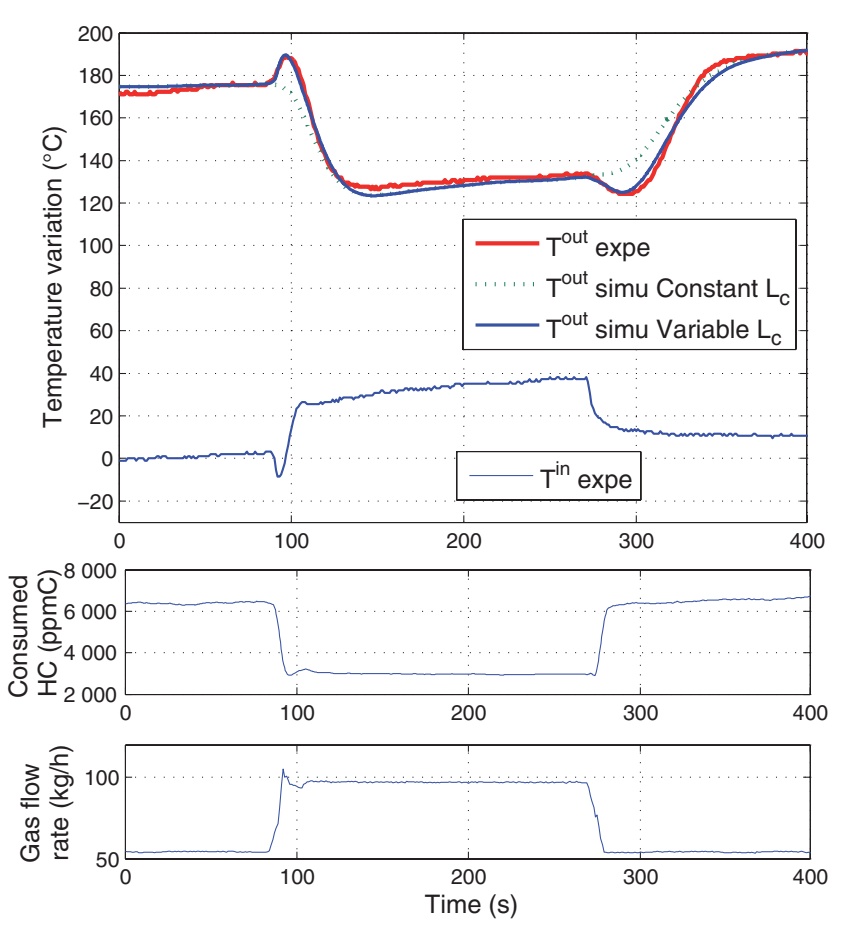

Figure 12

Model validation under variable gas flow. Accounting for variable reactive length reproduces the inverse response experimentally observed. Temperature variations $T(t)-T^{\text {in }}(0)$ are reported. Offset $T^{i n}(0)=412^{\circ} \mathrm{C}$. Consumed $\mathrm{HC}$ are estimated using inlet $\mathrm{HC}$ measurement and conversion efficiency (no outlet $\mathrm{HC}$ measurement). Dead time of the HC analyzer is accounted for in this figure.

DPF regeneration context since it increases the risk of DPF runaway. It is shown here how it can be compensated for.

To this purpose, a simple model is presented in Appendix A. This model, actually deduced from the reduced model [14], allows to describe the overshoot phenomena in its simplest form. The analytical study of this model gives the formal expression of the output response to a step change in $L_{c}(t)$, for an arbitrary trajectory, related to the actual control and forced to be uniform over the length $L_{c}(t)$. It is shown that, if the control is instantaneously changed to keep the same equilibrium output value, then the output response is subject to an overshoot. Then, a trajectory is proposed that limits the overshoot magnitude, but induces an undershoot. In view of real application it is not possible to use directly this trajectory in a controller. Indeed, it has been shown that the overshoot appears almost synchronous to the gas flow rate variation. This has been modeled by the phenomenological relation (7). In driving conditions, the trajectory of the gas flow rate is unknown. So, there is no other way to compensate for the overshoot but to reduce injection as soon as the gas flow rate variation 


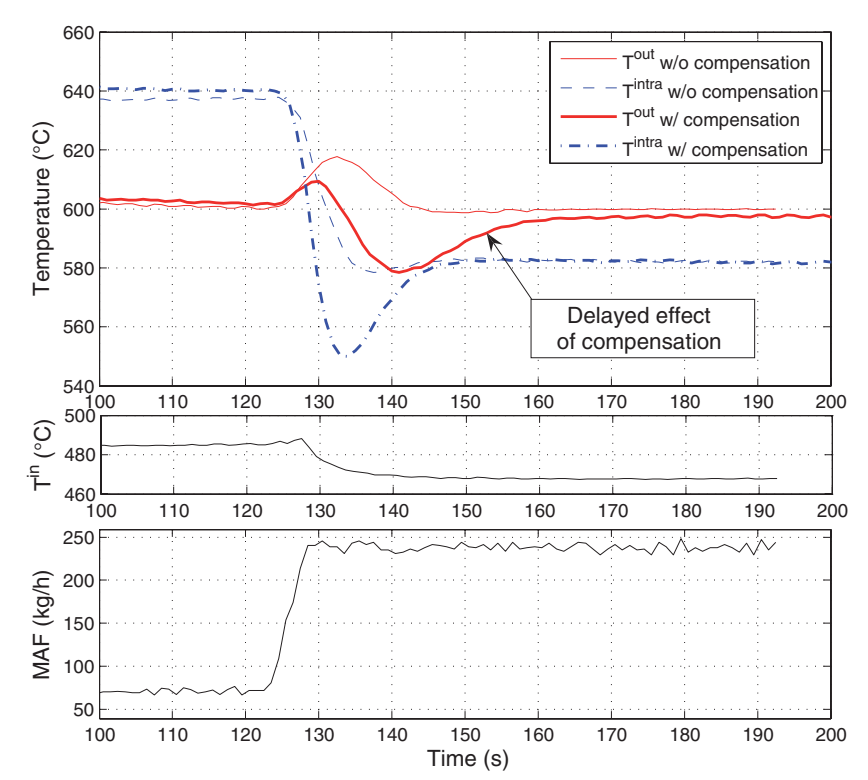

Figure 13

Overshoot attenuated by a reduction in late post-injection (experimental data).

is detected. To this end, an open-loop algorithm based on gas flow rate derivative is used in the following experiments to describe compensation possibilities in real applications. Using this algorithm, presented in Appendix B, leads to a causal control that closely imitates results of Appendix A.

In the experimental case presented in Figure 13, it can be seen that this reduction allows to attenuate the overshoot. It is interesting to notice that the consequence of this action is a delayed temperature undershoot (as marked in Fig. 13). This is an important result for control application since undershooting causes no risk of DPF runaway. Although not general, the case presented in Figure 14 is often encountered and has important consequences. It shows that the action of stopping late post-injection "only" attenuates the overshoot. The actuator (late post-injection) is stopped when the gas flow rate variation is detected. The detection is based on an algorithm using the filtered derivative of the gas flow rate. To ensure the algorithm robustness regarding unknown coming gas flow rate variations (in the perspective of control applications), the actuator Energizing Time (ET), is "only" reduced at the beginning of the gas flow rate variation, and totally cut off $(\mathrm{ET}=0)$ "only" about $2 \mathrm{~s}$ after gas flow rate starts varying. Upstream HC is measured using a slow gas analyzer, and its time constant must be accounted for while interpreting the results. Also, reductants flow rate related to the coupled post-injection (not controlled) may be large during the increase in engine power (producing the increase in the gas flow rate). This is why $\mathrm{HC}$ measurement decreases slowly.
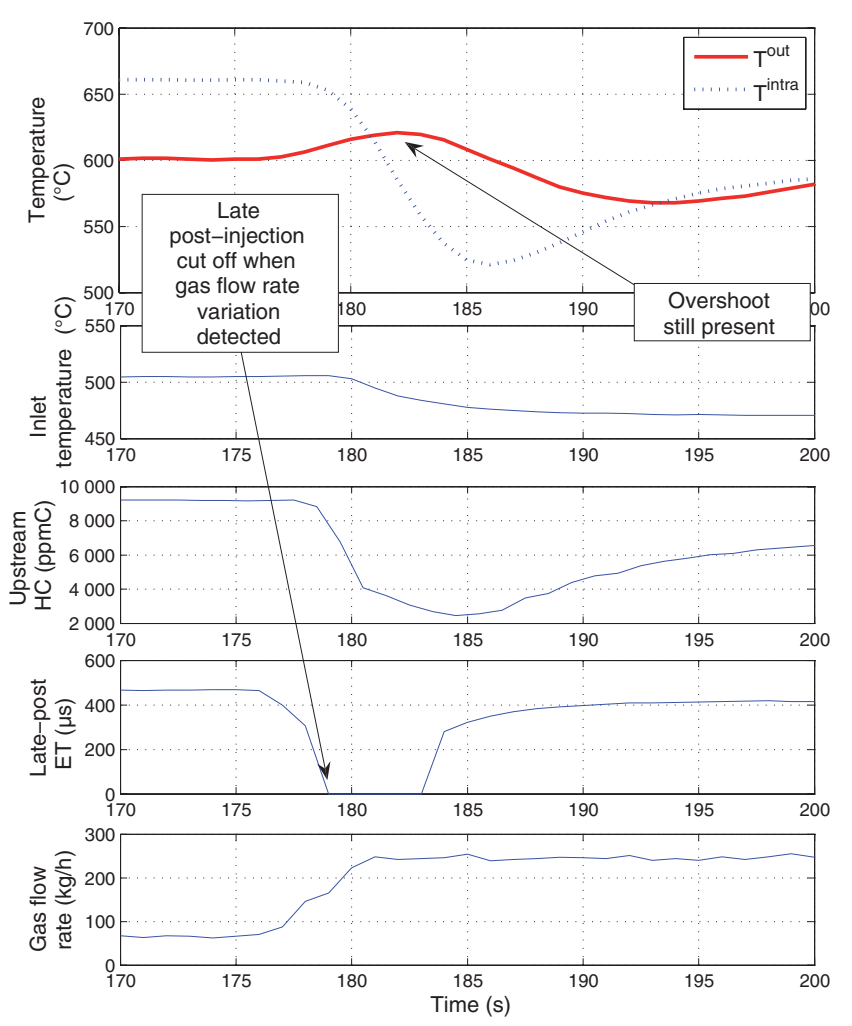

Figure 14

Overshoot is not totally compensated for by late post-injection cut-off (experimental data). Dead time of the HC analyzer is accounted for in this figure, but its time constant (slow analyzer) must be accounted for while interpreting the results.

\subsection{Compensation of Undershoot Phenomenon: a Risky Strategy}

The undershoot phenomenon has been introduced previously. It is related to a decrease in gas flow rate. Although it causes no risk of DPF damage, this variation is problematic in a DPF regeneration context. It increases the time required to oxidize the trapped soot and, in turn, fuel consumption. However, it will be shown that it cannot be compensated for without side effects.

To compensate for the undershoot, the reductant mass flow rate is increased. Compensation strategy is similar to one presented in Section 2.3 for the overshoot. Again, the algorithm of Appendix B is used. Here, a decrease in the gas flow rate leads to an increase in the reductant late post-injection. Two cases deserve to be detailed. First, with reasonable compensation, it is shown in Figure 15 that the undershoot is not totally compensated for. It is only reduced. It is worth noticing that this action causes a delayed temperature overshoot (as marked in Fig. 15). This delayed overshoot is caused by the transient increase in the postinjection - compared to the steady-state conditions - during 

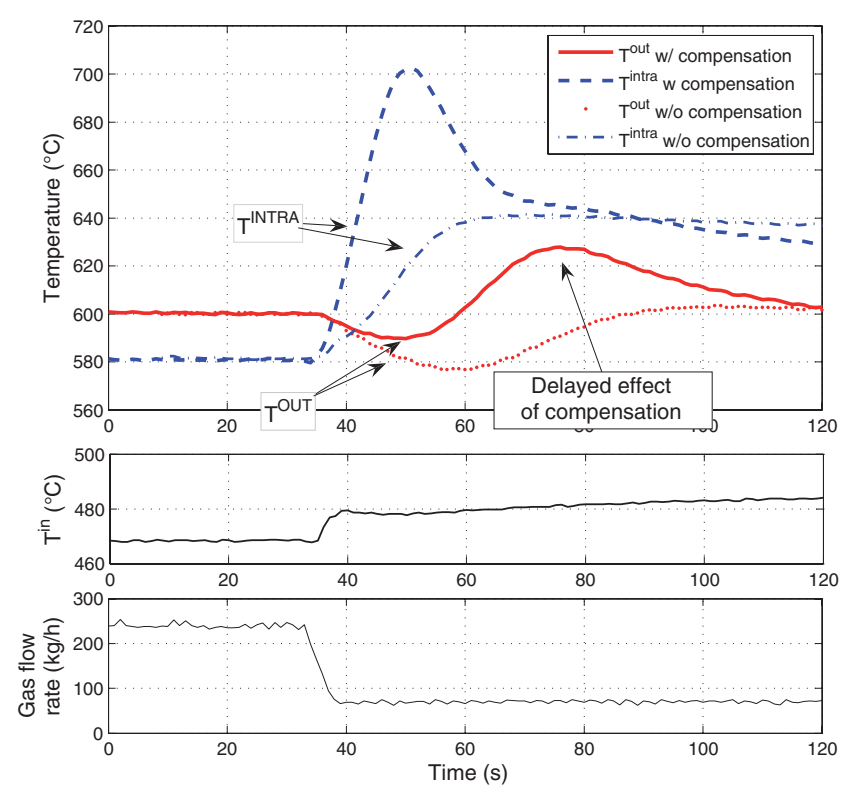

Figure 15

Small compensation for the undershoot (experimental data.) Upstream HC measurement is not available in this experiment. $\mathrm{HC}$ actuation is controlled by static mapping, which ensures the synchronization to the operating point. Downstream HC measurement (not plotted) show limited HC slip (normal operation).

the gas flow rate drop. Time between the gas flow rate variation and the delayed overshoot is related to phenomena described in Section 1.1. Referring to scheme Figure 11b, the compensation acts spatially on the reactive length $L_{c}$ during the transient state. On the contrary, the undershoot is related to the gap between the reactive length and the transport length. So, the compensation has no direct action on this gap: it acts always upstream. In fact, considering the involved time scales, the compensation effects and the supply gap effects are mixed by effects similar to diffusion. This explains why the undershoot is attenuated. From a practical viewpoint, the delayed overshoot is not suitable (for the reasons detailed in the previous subsection). It is also not easy to "re-compensate" for it, as it would imply an oscillatory highly-delayed control. Consequently, the undershoot must be carefully compensated for.

The second case is presented in Figure 16. Compared to the latter case, reductant quantity is increased further. It allows to completely compensate for the undershoot. Nevertheless, it must be noticed that the required amount of $\mathrm{HC}$ at the inlet is very large. Moreover, as shown in Figure 16, the amount of HC slipping to the outlet is also substantial. So, DOC has been saturated and the conversion efficiency has plummeted. Although more HCs are injected in the second case, the oxidized amount of $\mathrm{HC}$ is approximately identical

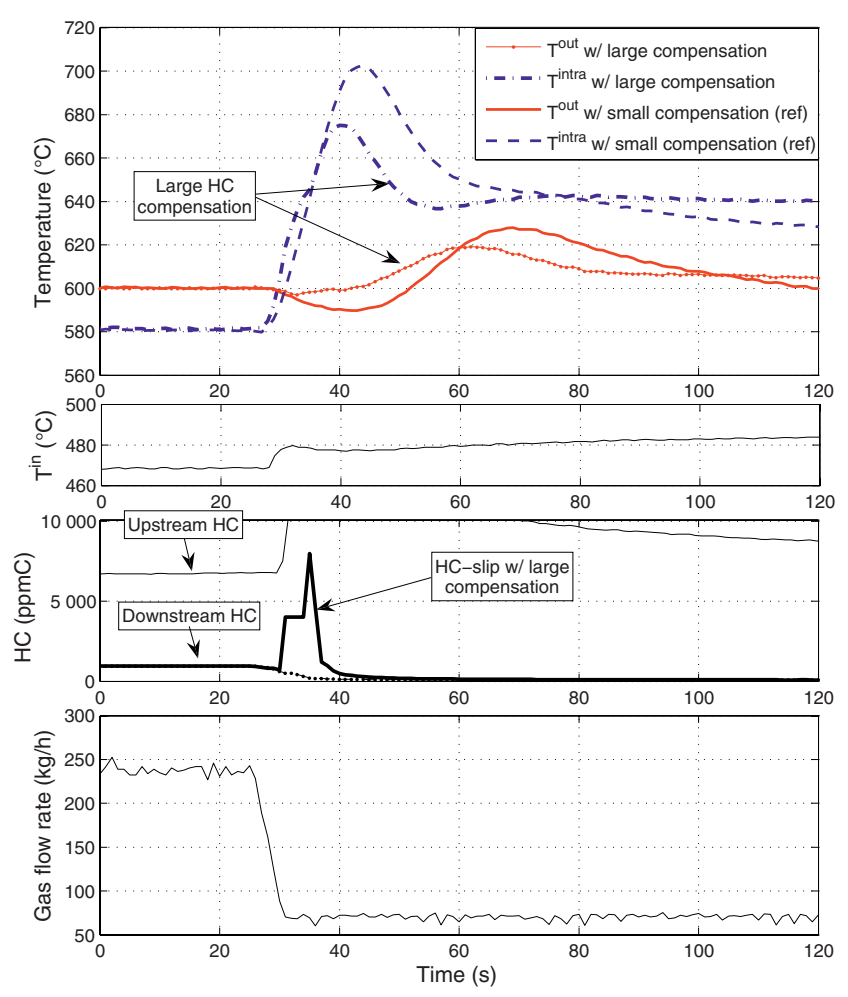

Figure 16

Large compensation for the undershoot (experimental data). Dead time of the $\mathrm{HC}$ analyzer is accounted for in this figure.

(it leads to identical inlet-to-outlet temperature rise). However, HCs are not oxidized in the same part of the DOC. In the second case, the proportion of $\mathrm{HC}$ oxidized in the vicinity of the outlet is greater than in the first case. In turn, between 30 and $50 \mathrm{~s}$, the intra-catalyst temperature rises less in the second case than in the first one, while outlet temperature rises more (no undershoot). Consequently to the intra catalyst temperature levels, the delayed outlet temperature overshoot, taking place between about 50 and $90 \mathrm{~s}$, is more important in the second case than in the first one. In fact, saturating the DOC forced the access to the $L_{c}$ variable, which is, under normal operation, given by the external conditions (by the gas flow rate in our modeling (see $E q .7$ ). In this way, it is possible to make up for the $L_{c}$ decrease induced by the decrease in gas flow rate. In other words, in the very special case of a huge level of inlet $\mathrm{HC}$, in spite of the decrease in gas flow rate, the phenomena must be described as pictured in Figure 17 (rather than Fig. 11b). Such a phenomenon could be easily included in the model presented in Section 1.7 by considering a growing $L_{c}$ length versus $\mathrm{HC}$ concentration. However, it is not useful to make the modeling more complex, because this "flooding" phenomenon must not be used in real applications. Saturations on the actuators must be implemented to prevent it. 


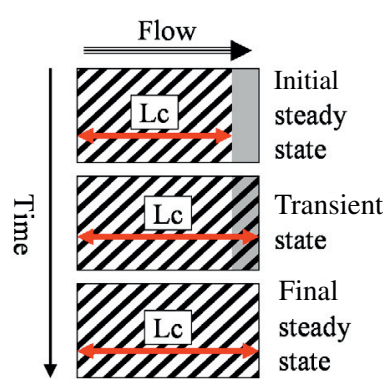

Figure 17

Phenomena involved in the special case of DOC saturation.

It has been shown that, although the compensation is physically possible, it clearly makes no sense to use it. As a conclusion, the undershoot phenomenon should not be compensated under normal operation.

\subsection{Conclusions}

The temperature overshoot, related to an increase in gas flow rate (at constant inlet $\mathrm{HC}$ concentration), can be, in some cases, experimentally compensated for. The temperature undershoot, related to a decrease in gas flow rate (at constant inlet $\mathrm{HC}$ concentration), can be experimentally compensated for but it causes high-level HC-slip. It should not be compensated for in practice. The presented observation aims to ground the conclusion on the limited control performance.

\section{CONCLUSIONS FOR DOC CONTROL APPLICATIONS}

This paper has focused on the rarely-described inverse response phenomena of the temperature variations during transients of gas flow rate (at constant inlet HC concentration). It has been shown how these phenomena can be easily modeled from a macroscopic viewpoint. The proposed model is supported by experimental results.

Based on this model, two different scenarios can be analyzed. In the case of an increase in the gas flow rate, the disturbance can be theoretically rejected. In the case of a decrease in the gas flow rate, the disturbance cannot be rejected under normal operation. However, it was shown experimentally that it was possible to reject the disturbance in the special case of "flooding" the DOC with HC. To avoid large quantities of HC-slip, this phenomenon is obviously problematic for practical applications. It must be prevented by saturating the actuators.

In automotive applications, the DOC can be used to raise the temperature of DPF during regenerations. Its outlet temperature must be carefully controlled. As a consequence of the described phenomena, desired performance for DOC outlet temperature control is physically limited. Depending on the tested DOC device, the expected range of performance would commonly not be better than $\pm 10^{\circ} \mathrm{C}$ to $\pm 20^{\circ} \mathrm{C}$ (or even worse).

The next step is to derive control laws from the presented simple model. It is worth studying how the non compensated effects can be taken into account in the feedback control law. The treatment of the other disturbances, particularly the inlet temperature and the engine $\mathrm{HC}$ emissions, must be considered as well.

\section{APPENDIX A: EXPLANATION OF OVERSHOOT ON A SIMPLE MODEL}

\section{A simple model to understand the origin of overshoot}

Following along a study in [14], it can be verified that the reduced model is equivalent, at constant speed $v(t)=v$ and at order 0 in $\varepsilon=v / k_{1}$, with $\vartheta=k_{1} v / k_{2}$, to

$$
\frac{\partial T(z, t)}{\partial t}+\vartheta \frac{\partial T(z, t)}{\partial z}=\Psi(z, u(t), v)
$$

Now, consider a length $L=1, \vartheta=1$ and $\Psi(z, u(t), v)=A(t)$ with

$$
\left\{\begin{array}{l}
A(t)=\alpha(t), \text { if } 0 \leq z \leq L_{c}(t) \\
A(t)=0, \text { if } L_{c}(t)<z \leq 1
\end{array}\right.
$$

To summarize, focusing on the advection effect, step changes in $L_{c}$ are analyzed on the following system

$$
\frac{\partial T(z, t)}{\partial t}+\frac{\partial T(z, t)}{\partial z}=A(t)
$$

\section{Existence of overshoot. Means to limit its magnitude}

For $T(0, t)=0, L_{c}(t)=L_{c, 1}$, and $\alpha(t)=\alpha_{1}$, the equilibrium is given by:

$$
T(z, t)= \begin{cases}\alpha_{1} z, & \text { if } 0 \leq z \leq L_{c, 1} \\ \alpha_{1} L_{c, 1}, & \text { if } L_{c, 1} \leq z \leq 1\end{cases}
$$

From this equilibrium, assuming that $\alpha(t)$ follows a trajectory such that $d \Gamma(t) / d t=\alpha(t)$, this yields, as $L_{c}(t)$ is stepped from $L_{c, 1}$ to $L_{c, 2}\left(L_{c, 2}>L_{c, 1}\right)$ at $t=0$ :

$$
\begin{aligned}
& T(1, t)= \\
& \begin{cases}\alpha_{1} L_{c, 1}, & \text { if } 0 \leq t \leq 1-L_{c, 2} \\
\alpha_{1} L_{c, 1}-\Gamma(0)+\Gamma\left(t-1+L_{c, 2}\right), & \text { if } 1-L_{c, 2} \leq t \leq 1-L_{c, 1} \\
\alpha_{1}(1-t)-\Gamma(0)+\Gamma\left(t-1+L_{c, 2}\right), & \text { if } 1-L_{c, 1} \leq t \leq 1 \\
\Gamma\left(t-1+L_{c, 2}\right)-\Gamma(t-1), & \text { if } t \geq 1\end{cases}
\end{aligned}
$$

The reader can refer to [14] for details of computations. 


\section{Simultaneous step change in $L_{c}(t)$ and $\alpha(t)$ : overshoot}

It is assumed that, as $L_{c}(t)$ changes from $L_{c, 1}$ to $L_{c, 2}\left(L_{c, 2} \geq\right.$ $\left.L_{c, 1}\right), \alpha(t)$ simultaneously changes from $\alpha_{1}$ to $\alpha_{2}$. To keep the same equilibrium value for $T(1, t), \alpha_{2}$ is chosen such that $\alpha_{1} L_{c, 1}=\alpha_{2} L_{c, 2}$. Then, $\alpha_{1} \geq \alpha_{2}$. In this case, $\Gamma(t)=\alpha_{2} t$ and (see the profile "without compensation"' in Fig. 19)

$$
\begin{aligned}
& T(1, t)= \\
& \begin{cases}\alpha_{1} L_{c, 1}, & \text { if } 0 \leq t \leq 1-L_{c, 2} \\
\alpha_{1} L_{c, 1}+\alpha_{2}\left(t-1+L_{c, 2}\right), & \text { if } 1-L_{c, 2} \leq t \leq 1-L_{c, 1} \\
\alpha_{1}(1-t)+\alpha_{2}\left(t-1+L_{c, 2}\right), & \text { if } 1-L_{c, 1} \leq t \leq 1 \\
\alpha_{2} L_{c, 2}, & \text { if } t \geq 1\end{cases}
\end{aligned}
$$

For $t$ from 0 to $1-L_{c, 2}$, the output is constant to its previous value $\alpha_{1} L_{c, 1}$. At $t=1-L_{c, 2}$, it begins to increase to reach its maximum $\alpha_{1} L_{c, 1}+\alpha_{2}\left(L_{c, 2}-L_{c, 1}\right)$ at $t=1-L_{c, 1}$. From that time, it decreases to reach its final value $\alpha_{2} L_{c, 2}$ at $t=1$. There is an overshoot of duration $L_{c, 2}$.

\section{Limiting the overshoot}

Now, consider that $\alpha(t)$ follows a ramp, from 0 at time 0 to $\alpha_{2}$ reached at $t=L_{c, 2}-L_{c, 1}$ (see trace "with compensation', in Fig. 18). From that time, $\alpha(t)=\alpha_{2}$ :

$$
\alpha(t)= \begin{cases}\frac{\alpha_{2}}{L_{c, 2}-L_{c, 1}} t, & \text { if } 0 \leq t \leq L_{c, 2}-L_{c, 1} \\ \alpha_{2}, & \text { if } t \geq L_{c, 2}-L_{c, 1}\end{cases}
$$

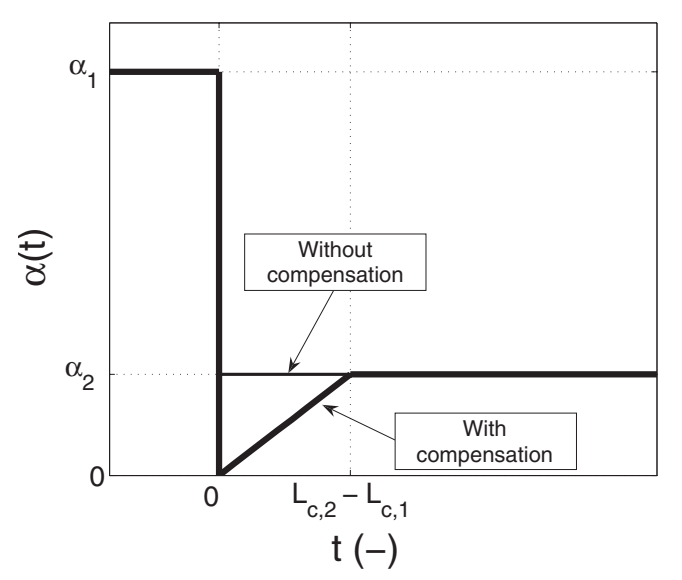

Figure 18

Profile of $\alpha(t)$ is plotted to illustrate the proposed compensation profile. Here, the length is changed from one constant value to another and the source term is updated to keep the same equilibrium point. Yet, during a period of time, an overshoot is observed at the outlet of the system (see Fig. 19).
Then, following computations details in [14],

$$
\Gamma(t)= \begin{cases}\frac{\alpha_{2}}{2\left(L_{c, 2}-L_{c, 1}\right)} t^{2}, & \text { if } 0 \leq t \leq L_{c, 2}-L_{c, 1} \\ \alpha_{2} t-\frac{\alpha_{2}\left(L_{c, 2}-L_{c, 1}\right)}{2}, & \text { if } t \geq L_{c, 2}-L_{c, 1}\end{cases}
$$

and

$$
\begin{aligned}
& T(1, t)= \\
& \left(\alpha_{1} L_{c, 1}, \quad \text { if } 0 \leq t \leq 1-L_{c, 2}\right. \\
& \alpha_{1} L_{c, 1}+\frac{\alpha_{2}}{2\left(L_{c, 2}-L_{c, 1}\right)}\left(t-1+L_{c, 2}\right)^{2} \text {, } \\
& \text { if } 1-L_{c, 2} \leq t \leq 1-L_{c, 1} \\
& \left\{\begin{array}{l}
\alpha_{1}(1-t)+\alpha_{2}\left(t-1+L_{c, 2}\right)-\frac{\alpha_{2}\left(L_{c, 2}-L_{c, 1}\right)}{2}, \\
\text { if } 1-L_{c, 1} \leq t \leq 1
\end{array}\right. \\
& \begin{array}{cc}
-\frac{\alpha_{2}}{2\left(L_{c, 2}-L_{c, 1}\right)}(t-1)^{2}+\alpha_{2}(t-1)+\frac{\alpha_{2}\left(L_{c, 1}+L_{c, 2}\right)}{2} \\
\text { if } 1 \leq t \leq 1+L_{c, 2}-L_{c, 1} \\
\alpha_{2} L_{c, 2}, \quad \text { if } t \geq 1+L_{c, 2}-L_{c, 1}
\end{array}
\end{aligned}
$$

For $t$ from 0 to $1-L_{c, 2}$, the output is constant to its previous value $\alpha_{1} L_{c, 1}$. At $t=1-L_{c, 2}$, it begins to increase to reach its maximum $\alpha_{1} L_{c, 1}+\alpha_{2}\left(L_{c, 2}-L_{c, 1}\right) / 2$ at $t=1-L_{c, 1}$. From that time, it decreases to reach the value $\alpha_{2}\left(L_{c, 1}+L_{c, 2}\right) / 2=$ $\alpha_{1} L_{c, 1}-\alpha_{2}\left(L_{c, 2}-L_{c, 1}\right) / 2$ at $t=1$. From $t=1$ it increases to reach its final value $\alpha_{2} L_{c, 2}$ at $t=1+L_{c, 2}-L_{c, 1}$. Refer to Figure 19. There is still an overshoot, but its maximum is divided by two. The counterpart is an undershoot that has the same magnitude than the overshoot. The total duration of the disturbance is increased by $L_{c, 2}-L_{c, 1}$.

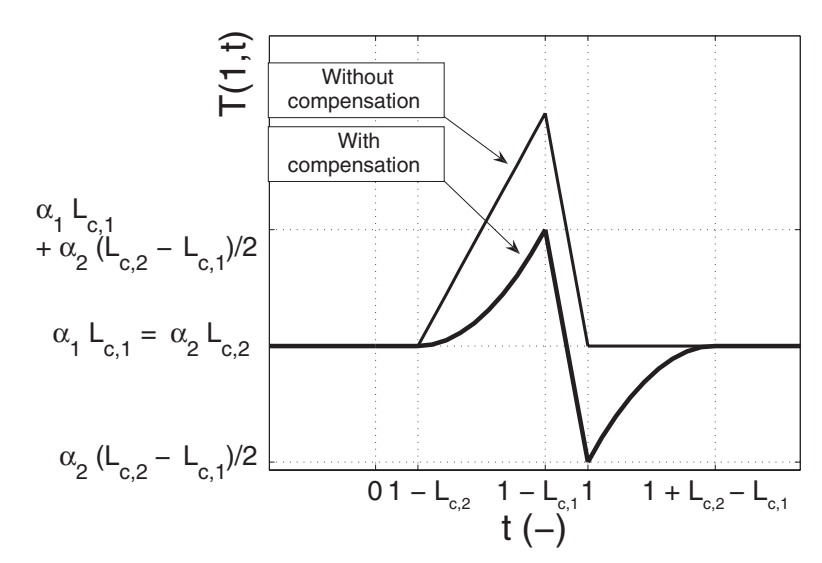

Figure 19

Profile of $T(1, T)$ is plotted to illustrate a partial overshoot compensation and the creation of a delayed undershoot. Compared to the case without compensation, the overshoot has been divided by two. 


\section{APPENDIX B: CAUSAL OPEN-LOOP COMPENSATION STRATEGY}

The simple advection model studied in Appendix A helps understand the overshoot phenomenon. The key aspect is the combination of heat storage and under-actuation, on a reactive length that is set by a disturbance, namely the gas flow rate. Means to limit the overshoot magnitude were also sketched. In view of real application, an easy-toimplement dynamic gain adaptation is used, based on the time derivative of the gas flow rate. It mimics the ramp variation described in Appendix A. A filtered derivative of the speed $v(t)$ is computed. The filter is a first order filter with time constant $\tau_{B}$. The filtered derivative is normalized with the current value of the gas flow rate, and multiplied by a gain $K_{B}$ to give a correction term $X$. The gain $G_{u}^{d y n}$ eventually used is given by $G_{u}^{d y n}=G_{u} /(1-X)$, where $G_{u}$ is the steady-state input-to-output gain relating a variation of the output temperature to the control variable. According to this dynamic correction, a rise (resp. decrease) of $v(t)$ induces a rise (resp. decrease) of $X$ : the value $G_{u}^{d y n}$ of the gain is then smaller (resp. larger) than the values of the static gain $G_{u}$. Accordingly, the control $u$ is less than what is required to keep $T^{\text {out }}$ constant. While $v(t)$ stabilizes to a new constant value, its derivative tends to 0 . Then, $X$ tends toward 0 and, as $G_{u}^{d y n}$ tends toward $G_{u}$, the control HC $u$ takes the value that is required to reach $T^{\text {out }}$ setpoint. In details,

$$
\frac{1}{G_{u}^{d y n}(t)}=\frac{1-X(t)}{G_{u}(t)}
$$

where $X(t)$ is given by $Y(t) / v(t), Y(t)$ corresponds to the inverse Laplace transform of $\hat{Y}(s)$ given by

$$
\hat{Y}(s)=\frac{K_{B} s}{1+\tau_{B} s} \hat{v}^{s a t}
$$

and $v^{\text {sat }}$ is the saturated value of the current gas speed. This saturation corresponds to the saturation of the length $L_{c}$ in Equation (7). $K_{B}$ is a tuning parameter corresponding to compensation strength. The greater $K_{B}$ is, the more the control HC flow is adapted during gas flow transients. If set too high, this parameter will favor delayed side effects. Besides, $\tau_{B}$ is a tuning parameter corresponding to a time during which the compensation acts after a gas flow rate variation. Different values of parameters $K_{B}$ and $\tau_{B}$ were explored. In the presented results, compensation action has been tuned to "off', (reference cases in Fig. 13 and 15), "medium', (compensated cases in Fig. 13 and 15), and "high" (Fig. 14 and 16).

\section{REFERENCES}

1 Ecopoint Inc. DieselNet. Available online: http://www. dieselnet.com/,February2008.

2 Bisset E.J. (1984) Mathematical model of the thermal regeneration of a wall-flow monolith diesel particulate filter, Chem. Eng. Sci. 39, 1232-1244.

3 Achour L. (2001) Dynamique et contrôle de la régénération d'un filtre à particules Diesel, $P h D$ Thesis, École des Mines de Paris - MINES ParisTech.

4 Koltsakis G.C., Stamatelos A.M. (1997) Catalytic automotive exhaust aftertreatment, Progr. Energ. Combust. Sci. 23, 1-39.

5 Depcik C., Assanis D. (2005) One-dimensional automotive catalyst modeling, Progr. Energ. Combust. Sci. 31, 308-369.

6 Oh S.H., Cavendish J.C. (1982) Transients of monolithic catalytic converters: response to step changes in feedstream temperature as related to controlling automobile emissions, Ind. Eng. Chem. 21, 29-37.

7 Lepreux O., Creff Y., Petit N. (2009) Model-based control design of a diesel oxidation catalyst, Proc. of ADCHEM 2009, International Symposium on Advanced Control of Chemical Processes, Istanbul, Turkey, July 2009.

8 Lepreux O., Creff Y., Petit N. (2008) Motion planning for a Diesel Oxidation Catalyst, Proc. of American Control Conference 2008, Seattle, USA, June 2008.

9 Lepreux O., Creff Y., Petit N. (2009) Warm-up strategy for a diesel oxidation catalyst, Proc. of European Control Conference 2009, Budapest, Hungary, Aug. 2009.

10 Petit N. (2010) Advances in the Theory of Control, Signals and Systems with Physical Modelling, Chapter Control Problems for One-Dimensional Fluids and Reactive Fluids with Moving Interfaces, pp. 323-337. Lecture Notes In Control and Information Sciences 407. Springer, 2010. ISBN: 978-3-642-16134-6.

11 Frobert A., Creff Y., Lepreux O., Schmidt L., Raux S. (2009) Generating Thermal Conditions to Regenerate a DPF: Impact of the Reductant on the Performances of Diesel Oxidation Catalysts, April 2009, SAE paper 2009-01-1085.

12 Osizik M.N. (1977) Basic heat transfer, McGraw-Hill.

13 Abramowitz M., Stegun I.A. (1965) Handbook of mathematical functions, Dover Publications, June 1965.

14 Lepreux O. (2009) Model-based Temperature Control of a Diesel Oxidation Catalyst, $P h D$ Thesis, École des Mines de Paris - MINES ParisTech. 